\title{
Catechin ameliorates depressive symptoms in Sprague Dawley rats subjected to chronic unpredictable mild stress by decreasing oxidative stress
}

\author{
AMITA RAI ${ }^{1,2^{*}}$, MEGHNA GILL ${ }^{1 *}$, MANAS KINRA $^{1}$, RAGHAVENDRA SHETTY $^{2}$, \\ NANDAKUMAR KRISHNADAS ${ }^{1}$, C. MALLIKARJUNA RAO ${ }^{1}$, SUHANI SUMALATHA ${ }^{3}$ and NITESH KUMAR ${ }^{1}$ \\ ${ }^{1}$ Department of Pharmacology, Manipal College of Pharmaceutical Sciences, \\ Manipal Academy of Higher Education; ${ }^{2}$ Ecron Acunova Ltd.; ${ }^{3}$ Department of Anatomy, \\ Kasturba Medical College, Manipal Academy of Higher Education, Manipal, Karnataka 576104, India
}

Received March 8, 2019; Accepted June 10, 2019

DOI: $10.3892 /$ br.2019.1226

\begin{abstract}
Catechin is an active ingredient of green tea. It is reported to inhibit corticosteroid-induced anxiety and depression-like symptoms. Considering the complex nature of depression, effects of catechin need to be studied in a clinically relevant depression model. The present study was designed to explore the antidepressant effect of catechin in Sprague Dawley rats subjected to chronic unpredictable mild stress (CUMS). Animals were subjected to CUMS and treated with $(+)$-catechin $(50 \mathrm{mg} / \mathrm{kg})$ or escitalopram $(10 \mathrm{mg} / \mathrm{kg})$ orally; a CUMS control and a vehicle control that was not exposed to CUMS were also established. Various stressors were applied daily in an unpredictable manner for 8 weeks achieve CUMS. Sucrose preference test were performed after 4 and 8 weeks and forced swim tests (FSTs) were conducted at weeks 4, 6 and 8 . At the end of week 8 , animals were sacrificed and the brain homogenate was studied for antioxidant parameters. Compared with the vehicle control, animals of the CUMS control group showed a significant decrease in sucrose intake. Catechin and escitalopram treatment significantly improved the sucrose intake compared with the CUMS control. A similar trend was observed in the FSTs, where catechin and escitalopram treatment significantly reduced the immobility time, and antioxidant parameters, including catalase, glutathione and superoxide dismutase levels were recovered in treated animals compared with the
\end{abstract}

Correspondence to: Dr Nitesh Kumar, Department of Pharmacology, Manipal College of Pharmaceutical Sciences, Manipal Academy of Higher Education, Madhav Nagar, Manipal, Karnataka 576104, India

E-mail: nitesh.kumar@manipal.edu

${ }^{*}$ Contributed equally

Key words: catechin, chronic unpredictable mild stress model, forced swim test, sucrose preference test
CUMS control. Thus, it was concluded that catechin reverses CUMS-induced depression in rats by ameliorating oxidative stress, which may help to develop a novel treatment for major depressive disorder.

\section{Introduction}

Depression is a mental health disorder, which varies from mild to severe changes in mood and affects physical, mental and behavior health (1). According to International Classification of Diseases-10 and Diagnostic and Statistical Manual-IV, depression episodes are recognized as an individual suffering from depressed or sad mood, showing a loss of energy, diminished activity or loss of interest in activities, which results in a significant reduction in productivity and has a negative impact on overall health (1). The World Health Organization projects unipolar major depression as a leading burden of disease worldwide by 2030 (2).

There are various factors that play a role in the development of major depression, such as changes in environmental factors, socioeconomic conditions, sedentary lifestyle, nutrition and eating habits (1). These conditions are linked to impacting stressful events in life. Continuous exposure to stress can cause a compensatory increase in synthesis and concentration of monoamines, specifically serotonin, norepinephrine and dopamine in brain (3), which are responsible for regulating appetite, the drive for sleep, emotions, stress and sexuality (3). Despite the adaptive consequence, changes in the nervous system can be excessive, leading to an increase in vulnerability to the pathology of depression (3). Chronic exposure to stressful events not only affects neurotransmitter levels but also induces neuroinflammation (4), increase oxidative stress (5) and affects the hypothalamic-pituitary-adrenal (HPA) axis (4). Changes in neurochemical levels can result in dampening the psychological or physical impact of stress and induce a response that impairs the ability in dealing with stress (6). Current treatments mainly target monoamines levels; however, this treatment is not efficient for many patients (7). An alternative approach is required to modulate neuroinflammation and resultant oxidative stress. 
Associations between oxidative stress and inflammatory cytokines are well established in major depressive disorders (5). Oxidative stress is an outcome of imbalance between oxidant and antioxidant levels that affects lipids and various other cellular biomolecules, and results in the generation of various inflammatory cytokines (5). It was reported that oxidative stress is elevated in major depressive syndrome and suppressed by standard monoamine-targeting drugs, such as amitriptyline, fluoxetine of imipramine (8). Thus, the present study was designed to target oxidative stress that resulted from chronic stress.

Diet is important in maintaining health. Green tea is a popular beverage consumed daily by a wide population; particularly in Japan and some parts of China, where $\leq 4$ cups are consumed daily (9). Its active ingredients, including (+)-catechin, (+)-catechin gallate, (-)-epicatechin, (-)-epicatechin gallate, (-)-epigallocatechin, (-)-epigallocatechin gallate, $(+)$-gallocatechin and (+)-gallocatechin gallate, have shown antioxidant, anti-inflammatory and neuroprotective effects in various animal models (10). Epigallocatechin-3-gallate, a catechin found in green tea, has shown catechol-o-methyltransferase inhibitory activity in vitro (11). Another active ingredient, (+)-catechin has shown anti-inflammatory effects in lipopolysaccharide-induced neuroinflammation (10). (+)-Catechin is an active constituent of the Tamarindus indica fruit pulp (12). Extract of it has shown aphrodisiac effect in the previous study, which is suggesting that catechin might alter monoamine levels in the brain (12). In chronic corticosterone-injected rats, catechin administration showed a decrease in depression and anxiety-like behaviors (13). However, these described corticosterone injections may not reflect stress-induced depression accurately. The chronic unpredictable mild stress (CUMS) model in rodents mimics responses similar to stressful life events in humans (14). Thus, the present study was designed to assess the antidepressant activity of (+)-catechin in rats with CUMS.

\section{Materials and methods}

Chemicals. Escitalopram oxalate (escitalopram; purity, >98\%) was provided by Micro Labs Ltd. and (+)-catechin hydrate, butylated hydroxytoluene (BHT), thiobarbituric acid (TBA), 5,5-dithio-bis-(2-nitrobenzoic acid) (DTNB) and adrenaline bitartrate were purchased from Sigma-Aldrich (Merck KGaA). Carboxymethyl cellulose (CMC) was purchased from Loba Chemie Pvt. Ltd., sodium chloride and trichloroacetic acid (TCA) were purchased from SD Fine-Chem Ltd. and hydrogen peroxide, sodium bicarbonate, potassium dihydrogen phosphate and disodium hydrogen phosphate were purchased from HiMedia Laboratories Pvt. Ltd.

Animals. A total of 24 male Sprague Dawley rats (age, 12 weeks; weight, $180-200 \mathrm{~g}$ ) were obtained from the Central Animal Research Facility of the Manipal Academy of Higher Education. Animals were acclimatized for 7 days before initiating the experiment. The housing, breeding and storage conditions were the same, with a controlled temperature $\left(20 \pm 5^{\circ} \mathrm{C}\right)$, humidity $(55 \pm 5 \%)$ and 12 -h light/dark cycles. Rats were kept in sterilized propylene cages containing sterile husk and were provided with food and water ad libitum.
The study was approved by the Institutional Animal Ethics Committee of Kasturba Medical College (Manipal, India; no. IAEC/KMC/49/2016). All the experiments were performed according to the Committee for the Purpose of Control and Supervision of Experiments on Animals guidelines (15).

Experimental design of CUMS. Rats were randomly divided into four groups ( $\mathrm{n}=6 / \mathrm{group}$ ): (i) Vehicle control, receiving $0.25 \%$ (w/v) CMC [dose volume, $10 \mathrm{ml} / \mathrm{kg}$ per os (p.o.)]; (ii) CUMS control, receiving $0.25 \%$ (w/v) CMC (dose volume, $10 \mathrm{ml} / \mathrm{kg}$ p.o.); (iii) escitalopram, receiving escitalopram $10 \mathrm{mg} / \mathrm{kg}$ p.o. as suspension in $0.25 \%$ (w/v) CMC (stock, $1 \mathrm{mg} / \mathrm{ml}$; dose volume, $10 \mathrm{ml} / \mathrm{kg}$ ) (14); and (iv) catechin, receiving (+)-catechin hydrate at $50 \mathrm{mg} / \mathrm{kg}$ p.o. as suspension in $0.25 \%$ (w/v) CMC (stock, $5 \mathrm{mg} / \mathrm{ml}$; dose volume, $10 \mathrm{ml} / \mathrm{kg}$ ) (16). All the rats were administered with their treatments daily between 9-10 am and $1 \mathrm{~h}$ prior to daily stress exposure for 8 weeks. All drug suspensions were prepared fresh on a daily basis in $0.25 \%$ CMC (14).

The CUMS procedure was performed as previously described (14). It involved the daily application of various stressors for a period of 8 weeks. The stressors included: (i) No food for $24 \mathrm{~h}$, (ii) no water for $20 \mathrm{~h}$, (iii) tail pinch for 30-60 sec, (iv) electric shock at $0.5 \mathrm{~mA}$ for $10-15 \mathrm{sec}$, (v) cage tilt at $45^{\circ}$ for $24 \mathrm{~h}$, (vi) restraint in a rat restrainer for 4-6 h, (vii) forced swimming for 15-20 min and (viii) no food and water for 20-24 h. They were applied following the schedule shown in Table I. Vehicle control rats were not exposed to any stressors.

Sucrose preference test. The sucrose intake study was performed as described previously (14) and involved an adaptation phase, where rats were adapted to consume $1 \%$ (w/v) sucrose before starting the CUMS experiment. In the next phase, the test phase, sucrose compared with water consumption was measured. During adaptation, rats were placed individually in a cage and provided with two bottles for 3 days, one with water and one with $1 \%$ sucrose solution. Sucrose consumption was measured every $24 \mathrm{~h}$ and a baseline value was obtained as the average consumed in 3 consecutive days. The tests were performed in weeks 4 and 8 , and food and water were removed for $24 \mathrm{~h}$ prior to the experiment. Then, two bottles were placed in each cage, one with water and one with $100 \mathrm{ml} 1 \%$ sucrose solution. After $24 \mathrm{~h}$, the consumed volume was measured in both bottles.

Forced swimming test (FST). Rats were individually placed in a water tank made of glass (height, $80 \mathrm{~cm}$; diameter, $30 \mathrm{~cm}$ ) at $23-25^{\circ} \mathrm{C}$. Experiments were performed in two sessions. In the first session, animals were trained to swim for $15 \mathrm{~min}$. The second session (test session) was conducted $24 \mathrm{~h}$ after the training session and $1 \mathrm{~h}$ post treatment. In this test session, rats were studied for immobility time for $10 \mathrm{~min}$. The study was conducted in weeks 4, 6 and 8 . Immobility was recorded as floating, with small movements or the head above the surface of the water. The behavior was recorded using a video camera and scored manually $(14,17)$.

Evaluation of antioxidant parameters. On completion of the study, rats were anaesthetized with intraperitoneal injections of ketamine $(87 \mathrm{mg} / \mathrm{kg})$ and xylazine $(13 \mathrm{mg} / \mathrm{kg})$. Once the 
Table I. Schedule for chronic unpredictable mild stress in rats

\begin{tabular}{|c|c|c|c|c|c|c|c|c|}
\hline Day & Week 1 & Week 2 & Week 3 & Week 4 & Week 5 & Week 6 & Week 7 & Week 8 \\
\hline 1 & $\mathrm{NF}$ & NF, ES & $\mathrm{ES}$ & $\mathrm{RS}, \mathrm{TP}$ & NW & ES & $\mathrm{CT}$ & $\mathrm{RS}$ \\
\hline 2 & NW & NF, TP & RS, TP & ES & $\mathrm{CT}$ & NF, TP & ES & $\mathrm{CT}$ \\
\hline 3 & $\mathrm{CT}$ & RS, TP & $\mathrm{CT}$ & NFW & $\mathrm{RS}$ & $\mathrm{CT}$ & $\mathrm{RS}$ & $\mathrm{ES}$ \\
\hline 4 & $\mathrm{TP}$ & $\mathrm{CT}$ & NF, TP & ES & NF, TP & NW, TP & $\mathrm{TP}$ & NW, TP \\
\hline 5 & $\mathrm{RS}$ & $\mathrm{ES}$ & NW, ES & $\mathrm{CT}$ & $\mathrm{ES}$ & $\mathrm{RS}$ & $\mathrm{ES}$ & $\mathrm{NF}$ \\
\hline 6 & ES & NFW & RS, TP & $\mathrm{FS}^{\mathrm{a}}, \mathrm{NFW}$ & RS, TP & $\mathrm{FS}^{\mathrm{a}}, \mathrm{NFW}$ & NF & $\mathrm{FS}^{\mathrm{a}}$, NFW \\
\hline 7 & FS & $\mathrm{RS}, \mathrm{TP}$ & FS & PT1 & $\mathrm{ES}$ & FST & NW & PT2 \\
\hline
\end{tabular}

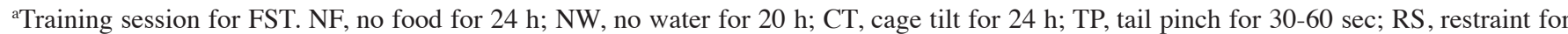
4-6 h; ES, electric shock for 10-15 sec; FS, forced swim for 15-20 min; NFW, no food and water for 20-24 h; PT, parameter testing (FST and SPT); FST, forced swim test; SPT, sucrose preference test.

animals reached the surgical plane of anesthesia, an intracardiac perfusion was given using PBS, pH 7.4 until the liver was clear. The brain was isolated and homogenated in PBS to make a $10 \%(\mathrm{w} / \mathrm{v})$ homogenate. The homogenate was centrifuged at $10,956 \mathrm{x}$ g for $30 \mathrm{~min}$ at $4^{\circ} \mathrm{C}$ and the supernatant was used to determine endogenous antioxidant levels, including catalase, reduced glutathione (GSH), superoxide dismutase (SOD) and malondialdehyde (MDA) using a UV spectrophotometer (18). Total protein was determined in the supernatant using Pierce ${ }^{\mathrm{TM}}$ BCA protein Assay kit (cat. no. 23225; Thermo Fisher Scientific, Inc.) as per manufacturer instruction.

Assay for antioxidant catalase levels. A total of $50 \mu \mathrm{l}$ tissue homogenate was added to $3 \mathrm{ml}$ of $50 \mathrm{mM}$ potassium phosphate buffer (PPB; pH 7.0) containing sufficient $\mathrm{H}_{2} \mathrm{O}_{2}(75 \mu 135 \%$ $\mathrm{H}_{2} \mathrm{O}_{2}$ added to $50 \mathrm{ml} \mathrm{PPB}$ ) to establish an OD between 3-5 at $240 \mathrm{~nm}$. Changes in absorbance were recorded for $1 \mathrm{~min}$. The activity of catalase was expressed as U/min/mg protein (19).

Assay for GSH levels. Protein was precipitated from $750 \mu 1$ tissue homogenate by adding an equal volume of $5 \%(\mathrm{w} / \mathrm{v})$ trichloroacetic acid. The supernatant was collected after centrifugation $\left(1,397 \times \mathrm{g}, 10 \mathrm{~min}, 4^{\circ} \mathrm{C}\right)$. A total of $500 \mu \mathrm{l}$ supernatant was added to a mixture of $3 \mathrm{ml} \mathrm{PB}(0.2 \mathrm{M}$; pH 8.0) and $500 \mu \mathrm{l}$ DTNB $(0.6 \mathrm{mM})$ and incubated at room temperature for $10 \mathrm{~min}$. Absorbance was recorded at $412 \mathrm{~nm}$. The amount of GSH was calculated by extrapolating the absorbance using a standard curve of GSH and was expressed in $\mu \mathrm{M} \mathrm{GSH} / \mathrm{mg}$ protein (19).

Assay of MDA levels. Tissue homogenate $(0.5 \mathrm{ml})$ was heated in a mixture of $2.5 \mathrm{ml} \mathrm{TBA}(0.375 \% \mathrm{w} / \mathrm{v})$, TCA $(15 \% \mathrm{w} / \mathrm{v})$ and BHT $(0.015 \% \mathrm{w} / \mathrm{v})$ solution at $90^{\circ} \mathrm{C}$ for $10 \mathrm{~min}$. The resulting mixture was centrifuged at $1,397 \mathrm{x}$ g for $10 \mathrm{~min}$ at $25^{\circ} \mathrm{C}$. The absorbance in the supernatant was directly recorded at $525 \mathrm{~nm}$. The absorbance was converted to the amount of MDA formed using the molar extinction coefficient of MDA and values were expressed in $\mathrm{nM} \mathrm{MDA} / \mathrm{mg}$ protein (19).

Assay for SOD levels. To $1.85 \mathrm{ml}$ carbonate buffer $(0.1 \mathrm{M}$; $\mathrm{pH} 10-11), 100 \mu \mathrm{l}$ adrenaline $(5 \mathrm{mM})$ and $50 \mu \mathrm{l}$ of tissue homogenate were added. Changes in the absorbance were recorded at $480 \mathrm{~nm}$ over $1 \mathrm{~min}$ at room temperature. The change in absorbance was extrapolated to a standard curve recorded for SOD activity. The activity was expressed in U/mg protein (19).

Statistical analysis. Data were analyzed using GraphPad Prism 5.0 (GraphPad Software, Inc.). Statistical comparisons were performed using one-way ANOVA followed by Tukey's multiple comparison tests. Data are expressed as the mean \pm standard error of the mean representative of six experimental repeats. $\mathrm{P}<0.05$ was considered to indicate a statistically significant difference.

\section{Results}

Catechin treatment affects FST in rats subjected to CUMS. CUMS control rats showed a significant increase in the immobility time in weeks 4,6 and 8 compared with the vehicle control group $(111.30 \pm 2.11,104.80 \pm 1.52$ and $115.50 \pm 0.76$ vs. $41.50 \pm 0.85$, $40.67 \pm 0.72$ and $41.33 \pm 1.12 \mathrm{sec}$, respectively; P<0.05; Fig. 1). Escitalopram treated animals subjected to CUMS displayed a significant reduction in immobility during the $10 \mathrm{~min}$ FST in weeks 4,6 and 8 , with $71.50 \pm 0.50,70.67 \pm 0.56$ and $69.83 \pm 0.70 \mathrm{sec}$, respectively, compared with the CUMS control group $(\mathrm{P}<0.05)$. This behavioral change indicated a reduction in the degree of despair/helplessness. Catechin treated animals subjected to CUMS also showed a significant reduction in immobility time compared with the CUMS control at the end of week 4, 6 and 8, with $77.33 \pm 1.09,76.83 \pm 0.95$ and $77.00 \pm 1.51 \mathrm{sec}$, respectively $(\mathrm{P}<0.05)$. The immobility time in the catechin treated group was found to be significantly higher compared with the escitalopram treated group in weeks 4, 6 and $8(\mathrm{P}<0.05)$.

Catechin treatment affects the consumption of sucrose water in rats subjected to CUMS. CUMS control animals showed a significant reduction in sucrose consumption compared with the vehicle control group observed at the end of week 4 and 8 $(\mathrm{P}<0.05)$. Additionally, in comparison with the baseline values recorded for the CUMS control group before subjecting the animals to CUMS, volumes of consumed sucrose decreased over the course of the experiment and exposure to CUMS, indicating anhedonia. Regular treatment of rats subjected to CUMS with escitalopram and catechin significantly increased sucrose consumption compared with the CUMS control group 

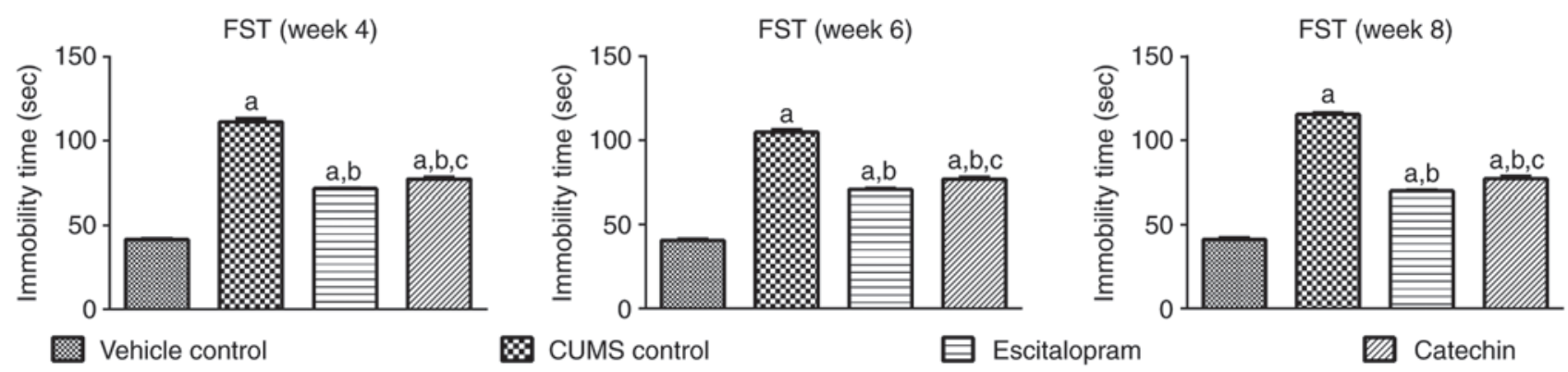

Figure 1. Catechin affects immobility time in FSTs. Rats were subjected to CUMS and treated with escitalopram or catechol from 8 weeks. At the end week 4 , 6 and 8 in rats underwent FSTs and the immobility times were recorded. ${ }^{\mathrm{P}} \mathrm{P}<0.05$ vs. vehicle control; ${ }^{\mathrm{b}} \mathrm{P}<0.05$ vs. CUMS control; and ${ }^{\mathrm{c}} \mathrm{P}<0.05$ vs. escitalopram. FST, forced swim test; CUMS, chronic unpredictable mild stress.

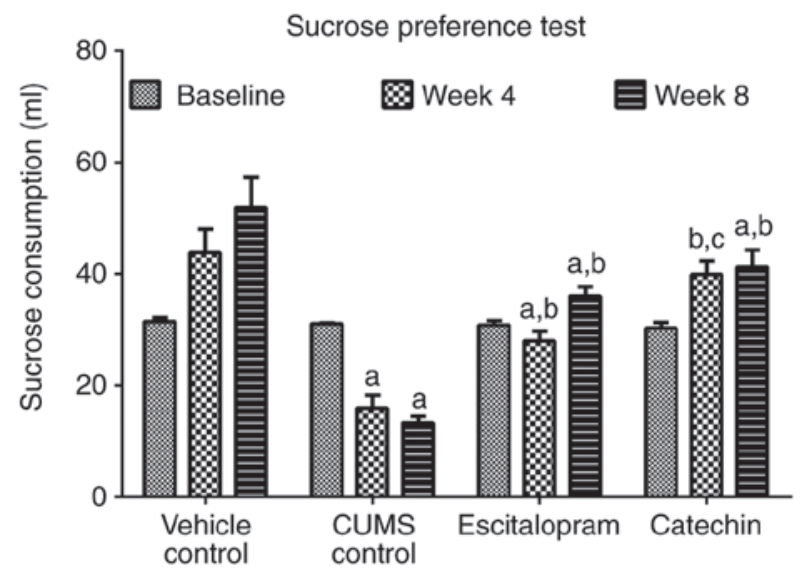

Figure 2. Catechin administration affects sucrose consumption in rats subjected to CUMS. Rats were subjected to CUMS and treated with escitalopram or catechol from 8 weeks. At week 4 and 8 , the preferential sucrose consumption was evaluated. ${ }^{a} \mathrm{P}<0.05$ vs. vehicle control; ${ }^{\mathrm{b}} \mathrm{P}<0.05$ vs. CUMS control; and ${ }^{\mathrm{C}} \mathrm{P}<0.05$ vs. escitalopram. CUMS, chronic unpredictable mild stress.

( $\mathrm{P}<0.05$; Fig. 2). In week 4, the sucrose consumption in the catechin treated group was significantly increased compared with the escitalopram treated animals $(\mathrm{P}<0.05)$.

Catechin treatment affects the endogenous antioxidant levels in the brain. The endogenous antioxidant activity, namely catalase and SOD activity, and reduced GSH and MDA levels were evaluated in brains of all animals after completion of the 8-week study (Fig. 3).

Catalase activity is recovered in catechin treated rats subjected to CUMS. In the CUMS control group a significant reduction in catalase activity was observed in comparison with the vehicle control group $(\mathrm{P}<0.05)$. Escitalopram and catechin treated animals subjected to CUMS showed a significant increase in catalase activity when compared with the CUMS control group $(\mathrm{P}<0.05)$.

Catechin affects SOD activity in rats subjected to CUMS. A significant reduction in SOD activity in the CUMS control group was observed in comparison with the vehicle control group $(\mathrm{P}<0.05)$. Escitalopram and catechin treated animals subjected to CUMS showed a significantly increased SOD activity in comparison with the CUMS control group $(\mathrm{P}<0.05)$.
Reduced GSH levels are recovered in catechin treated rats subjected to CUMS. A significant reduction in GSH levels in the CUMS control group was observed when compared with the vehicle control group $(\mathrm{P}<0.05)$. Regular treatment of animals subjected to CUMS with escitalopram or catechin significantly increased the levels of GSH compared with the CUMS control group $(\mathrm{P}<0.05)$. GSH activity in the catechin treated animals was significantly increased compared with the escitalopram treated animals $(\mathrm{P}<0.05)$.

Catechin treatment of rats subjected to CUMS reverses changes in MDA levels. A significant increase in MDA levels in the CUMS control group was observed when compared with the vehicle control group $(\mathrm{P}<0.05)$. Escitalopram and catechin treated animals subjected to CUMS showed significantly reduced levels of MDA compared with the CUMS control $(\mathrm{P}<0.05)$.

\section{Discussion}

The CUMS induced depression model is a suitable model for studying depression-like behavior in rodents. Chronic exposure to stress leads to behavioral and pathological alterations in rodents that are analogous to those observed in depressed patients (20). Chronic exposure to stress results in alterations in the central neurotransmitters, including changes in levels and activities of noradrenaline, serotonin and dopamine, in addition to the regulation of specific receptors, disruption of the HPA axis, elevation of cortisol levels leading to the activation of several proapoptotic factors, and the generation of reactive oxygen species (ROS) causing neurodegeneration and elevating the symptoms of depression (4). The present study proposed to assess the antidepressant activity of (+)-catechin in a rodent CUMS model of depression.

FST is a reliable behavioral test to assess depression in rodents (17). It helps in determining the degree of despair/helplessness, which is indicative of depression, and immobility observed in FST is considered analogous to depression in humans (14). FST is used to evaluate the activity of potential antidepressant drugs in rodent models of depression. The forced immersion of rodents in water for an extended time period leads to characteristic immobility. Antidepressant drugs decrease this immobility time. In the current study, treatment with catechin significantly reduced the FST immobility time at the end of week 4, 6 and 8 in animals subjected to CUMS 

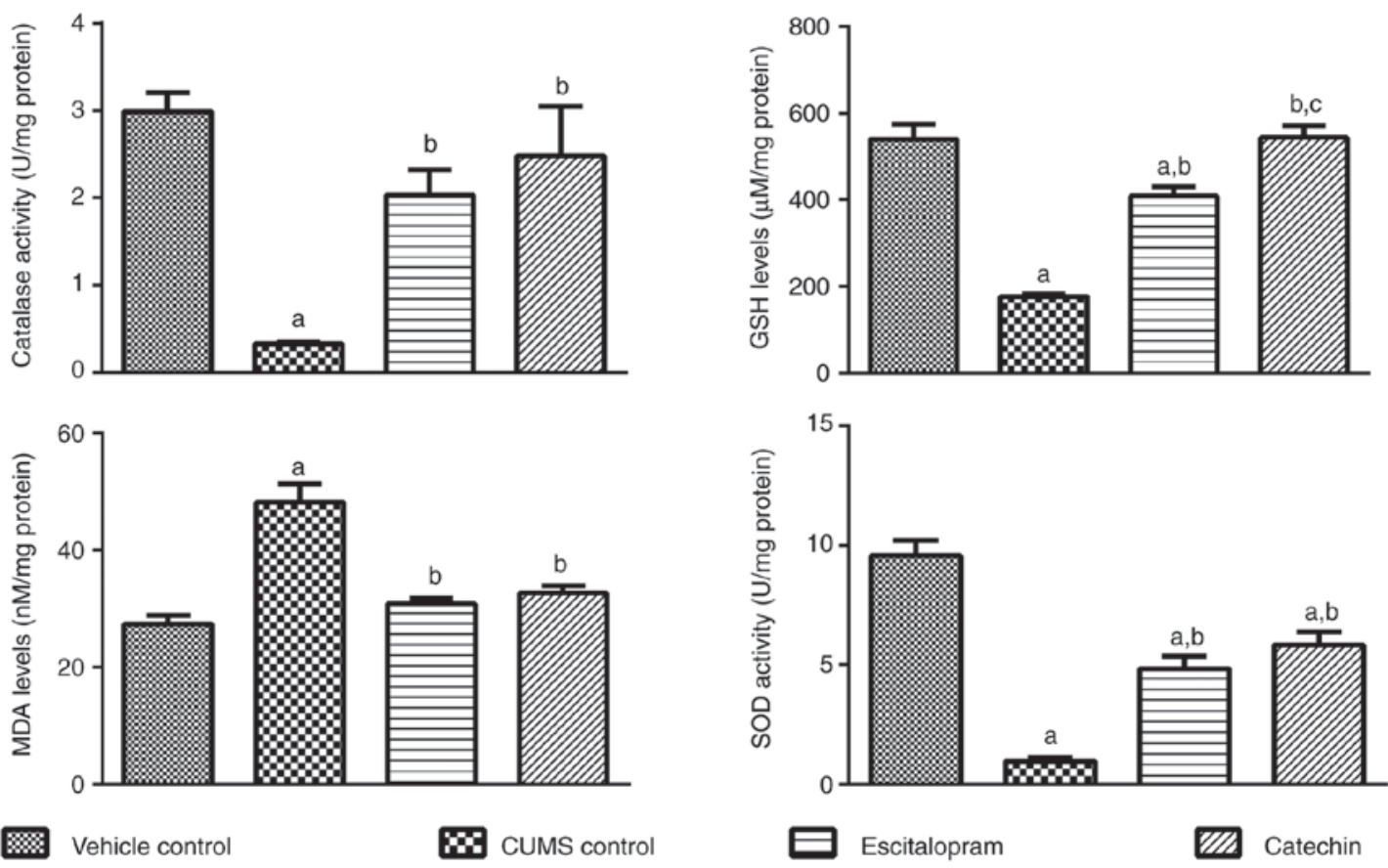

Figure 3. Catechin treatment in rats subjected to CUMS affects antioxidant levels in the brain. Rats were subjected to CUMS and treated with escitalopram or catechol from 8 weeks. After 8 weeks animals were sacrificed and the brains were collected for measurement regarding antioxidant levels; catechol and SOD activity, as well as GSH and MDA levels were determined. ${ }^{a} \mathrm{P}<0.05$ vs. vehicle control; ${ }^{\mathrm{b}} \mathrm{P}<0.05$ vs. CUMS control; and ${ }^{\mathrm{c}} \mathrm{P}<0.05$ vs. escitalopram. CUMS, chronic unpredictable mild stress; SOD, superoxide dismutase; GSH, glutathione; MDA, malondialdehyde.

compared with the CUMS control group, thereby indicating an antidepressant effect of the compound.

Anhedonia, or the lack of pleasure, is a major depressive symptom in humans (14). It can be assessed in rodents through a reduced preference for sucrose (14). In the current study, regular treatment of CUMS-subjected rats with catechin significantly increased the consumption of sucrose water compared with the CUMS control group after 4 and 8 weeks.

Oxidative stress is one of the main reasons for neurodegeneration (21) and for the further elevation of depression and its symptoms (22). Chronic exposure to stress results in the activation of several proapoptotic factors, the generation of excessive ROS and induces necrosis, thereby causing neurodegeneration, which serves a key role in the pathology of depression (5). The antioxidant capacity of the brain homogenate is an indicator of oxidative stress (5). Catalase is abundant in peroxisomes. Metabolic activity in cells leads to hydrogen peroxide production that can be toxic to the body causing the generation of ROS and inducing cell damage and death. Catalases catalyze the conversion of hydrogen peroxide to water and oxygen. Excessive oxidative stress leads to decreased catalase activity resulting in the accumulation of toxic hydrogen peroxide and ROS in the body (22). An increase in oxidative stress is reflected by a decrease in the levels of free radical scavengers, such as catalase, SOD and GSH, and an increase in the free radical generators, such as lipid peroxidation that is quantified through MDA levels (22). The present study showed that catechin significantly increased the levels of endogenous antioxidants in the brain, including catalase, SOD and GSH, and significantly decreased the levels of MDA, thereby preventing lipid peroxidation in the brain.

In the current study, antidepressant effects of catechin were compared with those of escitalopram, a known antidepressant. In week 4 , the immobility time in the escitalopram group was significantly decreased compared with the catechin group, while the sucrose consumption was significantly higher in the catechin group compared with the escitalopram group. There was no significant difference in oxidative stress indicators between the catechin and escitalopram groups except for the GSH activity. These results indicated that oxidative stress may not be the only target of catechin. Thus, it was suggested that regular treatment with catechin ameliorates the behavioral and neurochemical responses involved in the pathophysiology of depression due to its neuroprotective and antioxidant potential and the potential modulation of neurotransmitter and HPA activity, thus exhibiting antidepressant-like effects.

CUMS is associated with neuroinflammation and correlates with the brains' oxidative status (23). This mechanistic aspect was not further explored in the current study. The current study did not assess monoamine levels or changes of expression of proteins in the brain; these aspects may be explored in upcoming studies. Evaluating further underlying mechanisms in addition to the antioxidant axis may also be subject of future studies. Additionally, studies may assess molecular mechanisms, cortisol levels and mRNA expression in the brain and could help decoding the exact mechanism behind the antidepressant activity of catechin.

The outcomes of the present study supported ideas to use catechin as a nutritional supplement or as an adjuvant to the conventional antidepressant therapy to augment the effect of drugs (7). The antioxidant property exhibited by catechin, as well as its ability to modulate the levels of neurotransmitters may exert a long-term synergism with first-line drugs. However, further research is required to assess possible drug interactions in the co-administration with other drugs.

In conclusion, catechin showed prevention of anhedonia in Sprague Dawley rats subjected to CUMS that was observed 
by improvements in the sucrose preference test and reduced immobility times in FST. These observations were supported by a determined reduction in oxidative stress. Thus, it was suggested that catechin may be a novel therapeutic agent in treating stress-associated disorders, such as depression.

\section{Acknowledgements}

The authors like to thank Ms Ashwathi R. Hegde and Dr Muthukumar Amirthalingam (Department of Pharmaceutics, Manipal College of Pharmaceutical Sciences, Manipal Academy of Higher Education) for their support in this study in procuring chemicals. The authors further thank Mr. Raju Krishna Ekden and Mr. N. B. Shenoy from Micro Labs Ltd. for providing Escitalopram oxalate.

\section{Funding}

No funding was received.

\section{Availability of data and materials}

The datasets used and/or analyzed during the current study are available from the corresponding author on reasonable request.

\section{Authors' contributions}

AR, MG, RS, NaK, CMR and NiK designed the study, analyzed the data and drafted the manuscript. AR, MG and MK performed the in vivo behavioral study. SS, AR, MG and $\mathrm{NiK}$ performed the antioxidant analysis. All the authors read and approved the manuscript.

\section{Ethics approval and consent to participate}

Institutional Animal Ethics Committee of Kasturba Medical College approved the experimental procedures (approval no. IAEC/KMC/49/2016).

\section{Patient consent for publication}

Not applicable.

\section{Competing interests}

The authors declare that they have no competing interests.

\section{References}

1. Patel V: Cultural factors and international epidemiology. Br Med Bull 57: 33-45, 2001.

2. Lépine JP and Briley M: The increasing burden of depression. Neuropsychiatr Dis Treat 7 (Suppl 1): S3-S7, 2011.

3. Wang F and Pereira A: Neuromodulation, emotional feelings and affective disorders. Mens Sana Monogr 14: 5-29, 2016.

4. Slavich GM and Irwin MR: From stress to inflammation and major depressive disorder: A social signal transduction theory of depression. Psychol Bull 140: 774-815, 2014.

5. Lindqvist D, Dhabhar FS, James SJ, Hough CM, Jain FA, Bersani FS, Reus VI, Verhoeven JE, Epel ES, Mahan L, et al: Oxidative stress, inflammation and treatment response in major depression. Psychoneuroendocrinology 76: 197-205, 2017.
6. Anisman H, Merali Z and Hayley S: Neurotransmitter, peptide and cytokine processes in relation to depressive disorder: Comorbidity between depression and neurodegenerative disorders. Prog Neurobiol 85: 1-74, 2008.

7. Sarris J, Murphy J, Mischoulon D, Papakostas GI, Fava M, Berk M and $\mathrm{Ng} \mathrm{CH}$ : Adjunctive nutraceuticals for depression: A systematic review and meta-analyses. Am J Psychiatry 173: 575-587, 2016.

8. Behr GA, Moreira JC and Frey BN: Preclinical and clinical evidence of antioxidant effects of antidepressant agents: Implications for the pathophysiology of major depressive disorder. Oxid Med Cell Longev 2012: 609421, 2012.

9. Ni CX, Gong H, Liu Y, Qi Y, Jiang CL and Zhang JP: Green tea consumption and the risk of liver cancer: A meta-analysis. Nutr Cancer 69: 211-220, 2017.

10. Syed Hussein SS, Kamarudin MN and Kadir HA: (+)-catechin attenuates NF- $\kappa B$ activation through regulation of Akt, MAPK, and AMPK signaling pathways in LPS-induced BV-2 microglial cells. Am J Chin Med 43: 927-952, 2015.

11. Zhu BT, Shim JY, Nagai M and Bai HW: Molecular modelling study of the mechanism of high-potency inhibition of human catechol-O-methyltransferase by (-)-epigallocatechin-3-O-gallate. Xenobiotica 38: 130-146. 2008.

12. Rai A, Das S, Chamallamudi MR, Nandakumar K, Shetty R, Gill M, Sumalatha S, Devkar R, Gourishetti K and Kumar N: Evaluation of the aphrodisiac potential of a chemically characterized aqueous extract of Tamarindus indica pulp. J Ethnopharmacol 210: 118-124, 2018.

13. Lee B, Sur B, Kwon S, Yeom M, Shim I, Lee H and Hahm DH: Chronic administration of catechin decreases depression and anxiety-like behaviors in a rat model using chronic corticosterone injections. Biomol Ther (Seoul) 21: 313-322, 2013.

14. Gill M, Kinra M, Rai A, Chamallamudi MR and Kumar N: Evaluation of antidepressant activity of methanolic extract of Saraca asoca bark in a chronic unpredictable mild stress model. Neuroreport 29: 134-140, 2018.

15. Ministry of Environment and Forests and Climate Change, Government of India: Compendium of CPCSEA 2018. Committee for the Purpose of Control and Supervision of Experiments on Animals (CPCSEA), New Delhi, 2018. http://cpcsea.nic. in/WriteReadData/userfiles/file/Compendium\%20of\%20CPCSEA. pdf.

16. Zanwar AA, Badole SL, Shende PS, Hegde MV and Bodhankar SL: Chapter 21 - Antioxidant role of catechin in health and disease. In: Polyphenols in human health and disease. Watson RR, Preedy VR and Zibadi S (eds.) Academic Press, San Diego, CA, pp267-271, 2014.

17. Castagné V, Porsolt RD and Moser P: Use of latency to immobility improves detection of antidepressant-like activity in the behavioral despair test in the mouse. Eur J Pharmacol 616: 128-133, 2009.

18. Kumar N, Choudhary A, Tiwari A, Rasal A, Jetti R and Rao CM: Evaluation of hepatoprotective effect of aqueous extract of Schrebera swietenioides Bark against carbon tetrachloride-induced toxicity in wistar rats. Advanced Sci Lett 23: 1917-1920, 2017.

19. Kumar N, Rai A, Reddy ND, Shenoy RR, Mudgal J, Bansal P, Mudgal PP, Arumugam K, Udupa N, Sharma N and Rao CM: Improved in vitro and in vivo hepatoprotective effects of liposomal silymarin in alcohol-induced hepatotoxicity in Wistar rats. Pharmacol Rep (In Press).

20. Luo DD, An SC and Zhang X: Involvement of hippocampal serotonin and neuropeptide $\mathrm{Y}$ in depression induced by chronic unpredicted mild stress. Brain Res Bull 77: 8-12, 2008.

21. Cheruku S, Chamallamudi M, Ramalingayya G, Biswas S, Gourishetti K, Nandakumar K, Devkar R, Mallik SB, Nampoothiri M and Kumar N: Neuroprotective potential of methanolic extract of Saraca asoca bark against doxorubicin-induced neurotoxicity. Pharmacog Mag 15: 309-316, 2019.

22. Vaváková M, Duračková Z and Trebatická J: Markers of oxidative stress and neuroprogression in depression disorder. Oxid Med Cell Longev 2015: 898393, 2015.

23. Thakare VN, Patil RR, Oswal RJ, Dhakane VD, Aswar MK and Patel BM: Therapeutic potential of silymarin in chronic unpredictable mild stress induced depressive-like behavior in mice. J Psychopharmacol 32: 223-235, 2018. 